\title{
Formation of optical vortices with all- glass nanostructured gradient index masks
}

\section{Krzysztof Switkowski, Alicja Anuszkiewicz, Adam Filipkowski, Dariusz Pysz, Ryszard Stepien, et al.}

Krzysztof Switkowski, Alicja Anuszkiewicz, Adam Filipkowski, Dariusz Pysz, Ryszard Stepien, Wieslaw Krolikowski, Ryszard Buczynski, "Formation of optical vortices with all-glass nanostructured gradient index masks," Proc. SPIE 10712, Optical Manipulation Conference, 1071215 (24 April 2018); doi: 10.1117/12.2317053

SPIE. Event: SPIE Structured Light, 2018, Yokohama, Japan 


\title{
Formation of optical vortices with all-glass nanostructured gradient index masks
}

\author{
Krzysztof Switkowski, ${ }^{* a, b}$ Alicja Anuszkiewicz ${ }^{c}$, Adam Filipkowski ${ }^{c}$, Dariusz Pysz ${ }^{c}$, Ryszard \\ Stepien $^{\mathrm{c}}$, Wieslaw Krolikowski ${ }^{\mathrm{b}, \mathrm{d}}$, Ryszard Buczynski ${ }^{\mathrm{c}, \mathrm{e}}$ \\ ${ }^{a}$ Faculty of Physics, Warsaw University of Technology, Koszykowa 75, 00-662 Warsaw, Poland \\ ${ }^{\mathrm{b}}$ Science Program, Texas A\&M University at Qatar, Doha, Qatar \\ ${ }^{c}$ Department of Glass, Institute of Electronic Materials Technology, Wolczynska 133, 01-919, \\ Warsaw, Poland \\ ${ }^{\mathrm{d}}$ Laser Physics Centre, Research School of Physics and Engineering, Australian National University, \\ Canberra, ACT 0200, Australia \\ ${ }^{\mathrm{e}}$ Faculty of Physics, University of Warsaw, Pasteura 5, 02-093, Warsaw, Poland
}

\begin{abstract}
We report a development of microscopic size gradient index vortex masks using modified stack-and-draw technique. Vortex mask has a form of tens of microns thick, flat-surface all-glass plate. Its functionality is determined by internal nanostructure composed of two types of soft glass nanorods. Their spatial arrangement ensures that the average refractive index mimics continuous refractive index distribution imposing azimuthal phase modulation of optical beam. The mask of thickness of 40 microns is used to demonstrate generation of optical vortices with charges 1 and 2, in the femtosecond and $\mathrm{cw}$ regimes, respectively.
\end{abstract}

Keywords: optical vortices, singular optics, fibers, structured light, microstructured fibers

*Krzysztof.Switkowski@gatar.tamu.edu

\section{Introduction}

Optical vortices, i.e. optical beams with donut like intensity profile and helical (or screw-like) phase dislocation are subject of intense research into so called singular optics ${ }^{1}$.

Here we present for the first time generation of optical vortices by using gradient index phase masks fabricated by a modified stack-and-draw procedure similar to that employed in fabrication of microstructured fibers. Our approach is unique as it leads to flat surfaces, therefore the same component can be used in any medium as air or liquid.

\section{Mask design and fabrication}

The generation of optical vortex requires that the wave acquires an azimuthal phase change of $\Delta \varphi=2 \pi m$ with $m$ $=1,2 \ldots$ being integer. In the transmission geometry such a phase modulation can be achieved by helical variation of the thickness or refractive index of the medium. Here we are concerned with the latter approach. The required azimuthal phase variation requires continuous refractive index change $(\Delta \mathrm{n})$ such that the acquired total phase shift $\delta \varphi=\left(2 \pi / \lambda_{0}\right) \Delta \mathrm{n} d=2 \pi m$, where $\lambda_{0}$ denotes the wavelength in vacuum and $d$ is the thickness of the medium. To realize continuous variation of the refractive index we use, the so called, effective medium approach in which the medium consists of mixed nanosized regions of two materials with different refractive indices of the constituent materials.

(a)
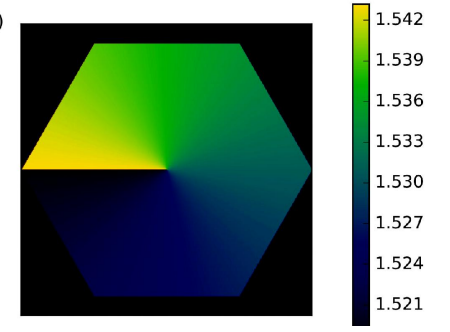

(b)

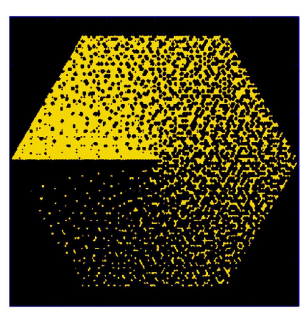

Figure 1. Refractive index distribution in gradient index (a) and nanostructured (b) vortex all-glass mask structure. Black and yellow regions in (b) correspond to low and high refractive index glass, respectively.

In our work we used nanorods made of two glasses with high and low refractive indices. Following the Maxwell

Optical Manipulation Conference, edited by Takashige Omatsu, Hajime Ishihara, Keiji Sasaki, Proc. of SPIE Vol. 10712, $1071215 \cdot$ • C 2018 SPIE · CCC code: 0277-786X/18/\$18 · doi: 10.1117/12.2317053 
Garnet formula and the effective medium theory ${ }^{2}$, the appropriate spatial arrangement of sub-wavelength nanorods, while discrete, will mimic continuous refractive index distribution. In fact we have already demonstrated fabricating flat gradient lenses and lens arrays ${ }^{3}$. In Figure 1(b) we show the discrete refractive index distribution which mimics the continuous one from Fig. 1(a). For fabrication of nanostructured vortex mask we used a modified stack-and-draw method commonly employed in photonic crystal fiber manufacturing ${ }^{4}$. We used nanorods made of two types of borosilicate glasses labeled NC21 and NC32. Their refractive indices at $\lambda=0.89 \mu \mathrm{m}$ are: $\mathrm{n}=1.518$ and $\mathrm{n}=1.543$, respectively.
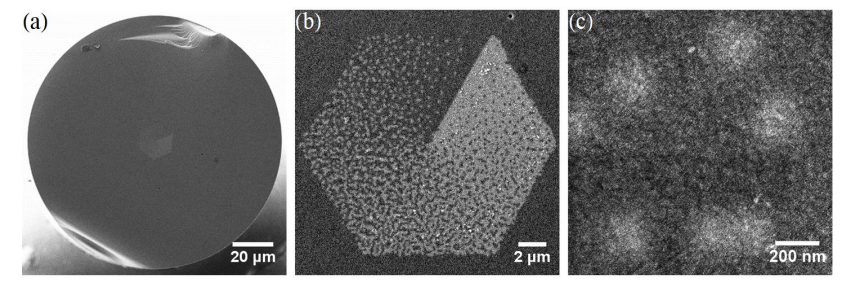

Figure 2. Fabricated vortex mask. (a) Scanning Electron Microscope (SEM) image of the end of the whole drawn fiber. Bright (dark) regions represent high (low) index glass. (b) SEM image of the vortex mask structure. (c) Magnified part of vortex structure with visible individual nanorods.

The hexagonal lattice in the preform is placed centrally in capillary made of low index glass NC21. The empty space between inner diameter of the capillary and nanostructured area is filled also with the low index NC21 rods. The preform has overall diameter of $55 \mathrm{~mm}$ and contains 101 glass rods. After initial assembly, the structure is drawn-down in a fibre-drawing tower to a rod with the diameter of $2 \mathrm{~mm}$ as shown in Fig. 2(c). The structured rod is then placed into a capillary to create intermediate preforms. After the drawing process we end up with a fiber $125 \mu \mathrm{m}$ in diameter, with the vortex structure positioned at the center having $19 \mu \mathrm{m}(16 \mu \mathrm{m})$ along the longer (shorter) diagonal. This final diameter corresponds to individual nanorod features of $190 \mathrm{~nm}$ making a fabricated component suitable for use under visible and near-IR illumination. The fiber is subsequently cut into slices, ground and polished such that the thickness of the final mask corresponds to desired vortex charge at a given wavelength. Fabrication of vortex with any charge is then straightforward task, since the mask is cut from a tens of meters long fiber.

\section{Generation of optical vortices}

The masks were optically tested by using both $\mathrm{cw}$ $\left(\lambda_{0}=532 \mathrm{~nm}\right)$ as well as short pulse laser beams $(680 \mathrm{~nm}$ to $1080 \mathrm{~nm}$ ). In the experiment the mask was glued to an edge of the $130 \mu \mathrm{m}$ microscope slide. The light beam was collimated and then focused with microscope objective $\times 10$ into a $12 \mu \mathrm{m}$ diameter spot at the center of the mask. The transmitted light was then collimated with microscope objective $\times 20$ and imaged onto the diffusive screen located at $60 \mathrm{~cm}$ distance from the mask. The resulting donut-like light intensity distribution was recorded with CCD camera. To determine the charge of the vortex we used two different techniques. For $\mathrm{cw}$ light, the vortex beam was superposed with the reference beam. This resulted in either fork or spiral intensity pattern for oblique or collinear interference, respectively. For short pulses we employed the known effect of vortex deformation by astigmatic transformation which results in vortex breakup and appearance of dark and bright extended stripes in the focal region ${ }^{5}$. The number of dark stripes indicates the charge of the vortex.

\section{Results and Discussion}

In Fig. 3 we show results of illumination of the vortex mask by femtosecond beam at $\lambda=890 \mathrm{~nm}$. Here the mask is $36 \mu \mathrm{m}$ thick. The graph in Fig. 3(a) depicts observed light intensity distribution of free propagating vortex beam. Its donut-like profile indicates singularity in the center. This is indeed confirmed by the astigmatic transformation of this beam induced by an angular misalignment of the imaging lens. It results in splitting of the vortex into two distinct bright stripes indicating the charge $m=1$. In the graphs Fig. 3(b) we shows results of numerical simulation of vortex generation by using exact discrete representation of the vortex mask as depicted in Fig. 1(b). Light intensity distribution 500 micrometers behind the mask is shown in top row of Fig. 3(b). The beam is clearly singular as confirmed by its helical phase structure (bottom row). Visible azimuthal nonuniformity of the beam reflects the influence of relatively long propagation through the mask. In order to minimize the deleterious propagation effect the mask should be shorter which can be achieved by using different types of glasses 

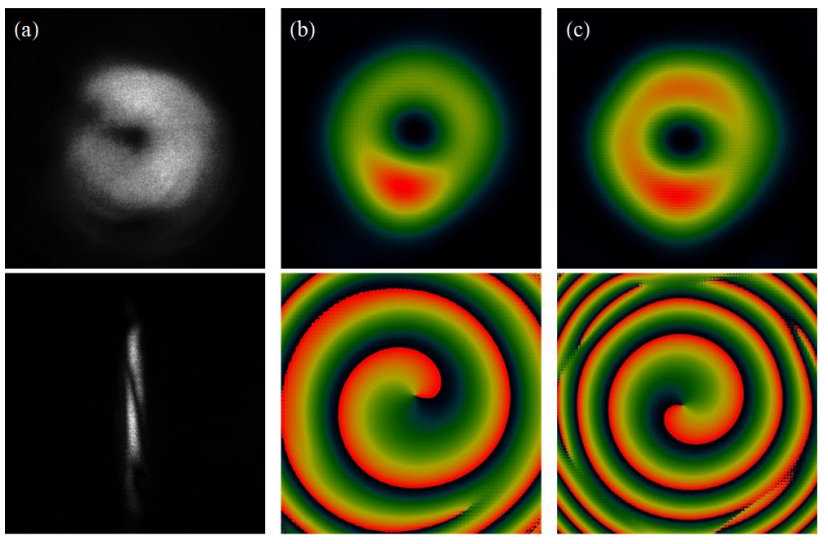

Figure 3. (a) Top: Light intensity distribution of the vortex formed after illuminating the mask with femtosecond laser beam at $\lambda_{0}=890 \mathrm{~nm}$. Bottom graph depicts the vortex intensity distribution after astigmatic transformation. Single dark stripe confirms charge $\mathrm{m}=1$ vortex. (b) calculated far field (top row) and phase (bottom row) of the beam generated by mask shown in Fig. 1(b), $500 \mu \mathrm{m}$ distance behind the mask. The mask thickness is $36 \mu \mathrm{m}$. (c) calculated far field intensity (top) and phase (bottom) of the $10.2 \mu \mathrm{m}$ mask formed using high contrast glasses.

with higher refractive index difference. This is demonstrated in Fig.3(c) which shows far field intensity (top) and phase structure (bottom) of the vortex beam generated from structured mask using higher refractive index contrast. In these simulation we used the NC21 glass $(\mathrm{n}=1.518)$ and commercial high-index lead-silicate F2 glass $(n=1.605)$. Clearly the quality of the generated vortex beam is improved.

In Fig. 4 we depict results for $\mathrm{cw} 532 \mathrm{~nm}$ beam. The first graph depicts again the characteristic donut-like intensity distribution. The corresponding interference patterns involving oblique [Fig. 4(b)] and collinear [Fig. 4(c)] reference wave show appearance of two extra fringes and two spirals indicating charge 2 vortex. This charge is also confirmed by the astigmatic transformation of the vortex beam [Fig. 4(d)] which results in appearance of two dark stripes in the focal plane. These observations agree with our calculations which show that at $\lambda_{0}=532 \mathrm{~nm}$ formation of a single charge vortex requires $20.7 \mu \mathrm{m}$ thick mask. Since our fabricated mask is roughly twice longer it generates charge 2 vortex.
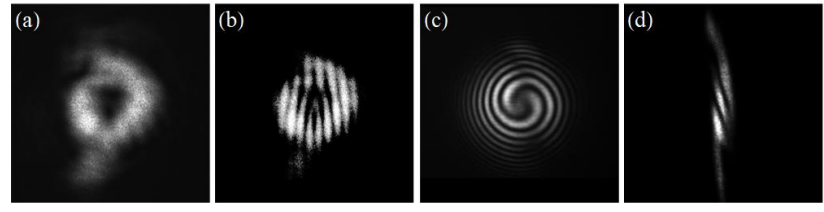

Figure 4. (a) Light intensity distribution of the vortex formed after illuminating the nanostructured vortex mask with focused cw $532 \mathrm{~nm}$ beam. The donut like intensity pattern indicates presence of phase singularity. (b,c) interferometric diagnostic of generated optical vortex by using tilted and collinear (c) reference beam. The presence of the fork having two more black line at the bottom than at the top of the fork structure and a double spiral pattern indicates charge of 2. (d) the light intensity of the vortex beam after astigmatic transformation by tilted lens.

In conclusion, we used a stack-and-draw technique to fabricate transmission flat phase mask for generation of optical vortices. We believe that these flat surface vortex masks of the diameter of tens of micrometers can be easily integrated into standard single and multimode fibers for potential application in fiber lasers, nonlinear microscopy and plasmonics.

Financial support: Qatar National Research Fund (NPRP8-246-1-060) and TEAM TECH/2016-1/1 (Foundation for Polish Science Team Programme from the funds of European Regional Development Fund under Smart Growth Operational Programme).

\section{References}

[1] D. L. Andrews, Ed. "Structured Light and Its Applications" (Elsevier, 2008).

[2] A. Sihvola, "Electromagnetic Mixing Formulas and Applications" (The Institution of Electrical Engineers, London, 1999).

[3] F. Hudelist, R. Buczynski, A. J. Waddie, and M. R. Taghizadeh, "Design and fabrication of nano structured gradient index microlenses," Opt. Express 17, 3255 (2009).

[4] D. Pysz, et al., "Stack and draw fabrication of soft glass microstructured fiber optics," Bull. Pol. Acad. Sci.-Tech. Sci., 62(4), 667-683 (2014).

[5] V. G. Shvedov, et al., "Efficient beam converter for the generation of high-power femtosecond vortices," Opt. Lett. 35, 2660 (2010) 